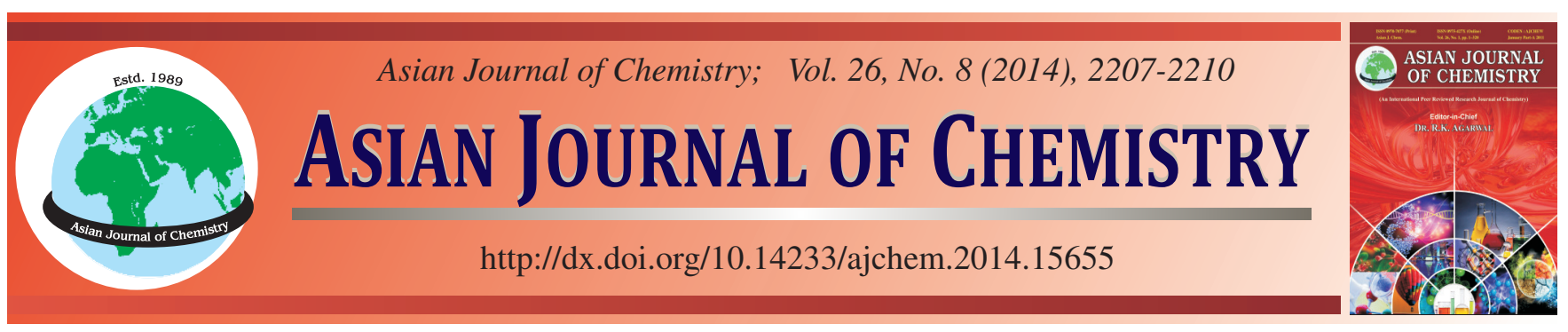

\title{
Chemical Variability of Citrus maxima Essential Oils from South India
}

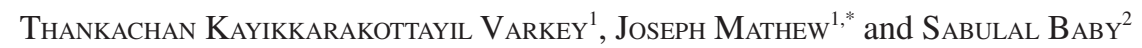

${ }^{1}$ Department of Chemistry, Mar Ivanios College, Nalanchira, Thiruvanthapuram-695 015, India

${ }^{2}$ Phytochemistry and Phytopharmacology Division, Jawaharlal Nehru Tropical Botanical Garden and Research Institute, Pacha-Palode, Thiruvananthapuram-695 562, India

*Corresponding author: E-mail: jmathewdr@gmail.com

Received: 18 April 2013;

Accepted: 24 August 2013;

Published online: 15 April 2014;

AJC-14993

Essential oils from the peels and leaves of Citrus maxima collected from five different geographical locations in south India were isolated by hydrodistillation. C. maxima peel and leaf oils were analyzed by the gas chromatography-mass spectrometry, constituents were identified by mass spectral database search, linear retention index data and comparison of mass spectra with literature. Twenty-two to twenty-five constituents (98.00-99.55\%) were identified in C. maxima peel oils. Major groups of compounds in C. maxima peel oils were monoterpene hydrocarbons (78.66-90.09\%), oxygenated monoterpenes (3.71-8.83\%) and sesquiterpene hydrocarbons $(3.86-10.51 \%)$. limonene (66.85-84.08\%), $\beta$-pinene (1.57-9.14\%), geranyl acetate (1.09-3.58 \%) and verbenone (0.27-3.00\%) were the major constituents in C. maxima peel oils. Twenty-nine to thirty-five constituents (97.90-99.15\%) were identified in C. maxima leaf oils. Monoterpene hydrocarbons, oxygenated monoterpenes, sesquiterpene hydrocarbons and oxygenated sesquiterpenes in C. maxima leaf oils were (14.0184.82, 6.47-50.41, 4.25-17.60 and 3.10-30.00\%), respectively. Limonene contents in C. maxima leaf oils were 5.29 to $78.45 \%$, with highest in the pathanamthitta accession. Other major constituents in C. maxima leaf oils were $\alpha$-pinene (t-21.09\%) and $\beta$-pinene (t-16.93 $\%)$. Limonene with sesquiterpenes and their oxygenated derivatives in relatively minor quantities are the constituents giving flavour and aroma to $C$. maxima peel and leaf oils. Only limited variability was observed between the chemical profiles of the five $C$. maxima accessions from south India.

Keywords: Citrus maxima, Chemical variability, Limonene, Geranyl acetate, Verbenone, $\alpha$-Pinene, $\beta$-Pinene.

\section{INTRODUCTION}

Citrus maxima (J. Burman) Merrill (Citrus grandis (L.) Osbeck, Rutaceae) is a medium sized tree $(5-15 \mathrm{~m})$ native to Asia. Its leaves have small winged petioles. It is commonly known as shaddock or pomelo. In traditional medicine $C$. maxima fruit peel is used for cough, swelling and epilepsy ${ }^{1}$. Citrus species are well known sources of the essential oils ${ }^{2,3}$. C. maxima and other Citrus species have essential oil glands in their fruit peel, leaves and petals ${ }^{4}$. Chemical compositions of Citrus essential oils depend on variations in environmental conditions, collection period, isolation methods, dehydration procedure and storage conditions ${ }^{5}$. Examples of previous studies on chemical profiles of $C$. maxima volatile oils (collection location, plant part, distillation method, analysis techniques, major constituents): (i) Thailand, peel and flower, super critical carbon dioxide extraction, GC-MS, peel oil, limonene $93.74 \%$, myrcene $1.71 \%$, germacrene D $1.04 \%$ and flower oil, limonene $86.2 \%$, (E)- $\beta$-ocimene $2.85 \%$, myrcene $1.77 \%$, geranial 1.17 $\%^{6}$, (ii) Vietnam, peel, cold pressing method, GC, GC-MS, limonene $98.7 \%$. Nootkatone was found in trace amounts ${ }^{7}$, (iii) Kenya, peel, cold pressing method, GC, GC-MS, limonene $91.10 \%, \beta$-caryophyllene $4.20 \%, \alpha$-cubebene $2.00 \%{ }^{2}$, (iv) China, peel, steam distillation, GC, GC-MS, limonene 62.48 $\%$, anethole $9.50 \%$, nootkatone $5.60 \%$, linalool $2.30 \%, \beta$ caryophyllene $2.26 \%$, (v) Tunisia, peel (four cultivars), GC, GC-MS, limonene $92.52-97.3 \%$, $\beta$-pinene $1.37-1.82 \%$. The observed chemical variability between these four cultivars is mainly due to the influence of the different environmental factors ${ }^{9}$, (vi) Japan, peel, cold pressing method, GC, GC-MS, limonene $87.07 \%$, $\alpha$-terpinene $6.04 \%$, myrcene $1.81 \%, \alpha$ pinene $1.13 \%^{10}$. The quality and the odor of $C$. maxima volatile oils are influenced by their limonene contents. There are no systematic reports on the essential oil compositions of $C$. maxima from southern India. Here, we report the comparative chemical profiles of volatile oils isolated from the peels and leaves of C. maxima collected from five geographical locations in south India.

\section{EXPERIMENTAL}

C. maxima fruits (peels) and leaves were collected during the October to December 2009 from five locations 
(i) Thiruvananthapuam, Kerala (CM-TH-1), (ii) Kottayam, Kerala (CM-KT-2), (iii) Pathanamthitta, Kerala (CM-PT-3), (iv) Alappuzha, Kerala (CM-AL-4) and (v) Kanyakumari, Tamil Nadu (CM-TN-5).

Oil isolation: Essential oils from fresh peels and leaves of five $C$. maxima accessions were isolated separately by hydrodistillation for $5 \mathrm{~h}$ on a Clevenger apparatus. Pleasant smelling, transparent, yellow coloured oils were obtained from the peels and leaves of C. maxima. CM-TH-1: peels $250 \mathrm{~g}$, oil yield $1.7 \mathrm{~mL}(0.68 \%$, v/w); leaves $250 \mathrm{~g}$, oil yield $0.2 \mathrm{~mL}$ $(0.08 \%, \mathrm{v} / \mathrm{w})$; CM-KT-2: peels $400 \mathrm{~g}$, oil yield $3.12 \mathrm{~mL}$ $(0.78 \%$, v/w); leaves $280 \mathrm{~g}$, oil yield $0.31 \mathrm{~mL}(0.11 \%$, v/w); CM-PT-3: peels $425 \mathrm{~g}$, oil yield $1.50 \mathrm{~mL}(0.35 \%$, v/w); leaves $300 \mathrm{~g}$, oil yield $0.31 \mathrm{~mL}(0.10 \%$, v/w); CM-AL-4: peels $450 \mathrm{~g}$, oil yield $1.3 \mathrm{~mL}(0.28 \%$, v/w); leaves $320 \mathrm{~g}$, oil yield 0.31 $\mathrm{mL}(0.09 \%, \mathrm{v} / \mathrm{w})$; CM-TN-5: peels $430 \mathrm{~g}$, oil yield $1.9 \mathrm{~mL}$ $(0.44 \%$, v/w); leaves $350 \mathrm{~g}$, oil yield $0.46 \mathrm{~mL}(0.13 \%$, v/w). C. maxima leaf and peel oils were stored at $4{ }^{\circ} \mathrm{C}$ until further analysis.

GC-MS analyses: GC-MS analyses of C. maxima peel and leaf oils were carried out by splitless injection of $1 \mu \mathrm{L}$ of each oil on a Agilent 6890 gas chromatograph (HewlettPackard, USA) fitted with an HP-5 (5\% phenyl $95 \%$ dimethyl polysiloxane, non-polar, $30 \mathrm{~m} \times 0.32 \mathrm{~mm}$ i.d., $0.25 \mu \mathrm{m}$ film thickness) capillary column, coupled with a Model 5973 mass detector. GC-MS operation conditions: injector temperature $220^{\circ} \mathrm{C}$; transfer line $240{ }^{\circ} \mathrm{C}$; detector temperature $250{ }^{\circ} \mathrm{C}$; oven temperature programme $60-246{ }^{\circ} \mathrm{C}\left(3{ }^{\circ} \mathrm{C} / \mathrm{min}\right)$; carrier gas $\mathrm{He}$ $1.4 \mathrm{~mL} / \mathrm{min}$; mass spectra-electron impact $\left(\mathrm{EI}^{+}\right)$mode $70 \mathrm{eV}$; ion source temperature $240{ }^{\circ} \mathrm{C}$.

Identification of oil constituents: Linear retention indices of oil constituents in Tables 1 and 2 were determined on the HP-5 column, using standard $\mathrm{C}_{5}-\mathrm{C}_{30}$ straight chain hydrocarbons (Aldrich Chemical Company, USA). Individual compounds in C. maxima peel and leaf oils were identified by Wiley $275 \mathrm{~L}$ database matching, comparison of mass spectra with published data and by comparison of their LRIs ${ }^{11}$ (Tables 1 and 2).

\section{RESULTS AND DISCUSSION}

Peels and leaves of five C. maxima accessions from south India on hydrodistillation yielded $0.28-0.78$ and $0.08-0.13 \%$ (v/w) of volatile oils, respectively. Both peel and leaf oils were pleasant smelling, yellow coloured and transparent. Twentytwo to twenty-five constituents (98.00-99.55\%) of C. maxima peel oils were identified by GC-MS analyses (Table-1). Monoterpene hydrocarbons (78.66-90.09\%) were the major class of compounds in all five accessions of C. maxima peel oils, followed by sesquiterpene hydrocarbons (3.86-10.51\%) and oxygenated monoterpenes (3.71-8.83\%). Limonene was the single major constituent in C. maxima peel oils (66.85$84.08 \%$ ) and it gives the pungent smell to these volatile oils. $\beta$-Pinene (5.15-9.14\%), geranyl acetate (1.09-3.58 \%) and verbenone $(0.27-3.00 \%)$ were the other major terpenoids in

TABLE-1

COMPARATIVE CHEMICAL PROFILES OF THE PEEL ESSENTIAL OILS OF FIVE Citrus maxima ACCESSIONS FROM SOUTH INDIA

\begin{tabular}{|c|c|c|c|c|c|c|c|}
\hline Constituent & $\begin{array}{l}\text { LRI } \\
\text { (cal.) }\end{array}$ & $\begin{array}{l}\text { LRI } \\
\text { (lit.) }\end{array}$ & $\begin{array}{c}\text { CM-KT-1 } \\
(\%)\end{array}$ & $\begin{array}{c}\text { CM-TH-2 } \\
(\%)\end{array}$ & $\begin{array}{c}\text { CM-PT-3 } \\
(\%)\end{array}$ & $\begin{array}{c}\text { CM-AL-4 } \\
(\%)\end{array}$ & $\begin{array}{c}\text { CM-TN-5 } \\
(\%)\end{array}$ \\
\hline$\beta$-Pinene & 974 & 974 & 9.14 & 7.63 & 5.15 & 7.36 & 1.57 \\
\hline cis-meta-Mentha-2,8-diene & 984 & 983 & 0.63 & $\mathrm{t}$ & $\mathrm{t}$ & 0.61 & $\mathrm{t}$ \\
\hline Limonene & 1027 & 1024 & 66.85 & 81.42 & 84.08 & 80.11 & 79.78 \\
\hline$\beta$-Ocimene & 1046 & 1044 & $\mathrm{t}$ & $\mathrm{t}$ & 0.86 & $\mathrm{t}$ & $\mathrm{t}$ \\
\hline$\gamma$-Terpinene & 1054 & 1054 & 2.04 & 0.14 & $\mathrm{t}$ & 1.45 & 0.36 \\
\hline Linalool oxide & 1066 & 1067 & 1.15 & 2.78 & $\mathrm{t}$ & 0.91 & $\mathrm{t}$ \\
\hline iso-Terpinolene & 1081 & 1085 & 0.51 & t. & 0.24 & 0.25 & $\mathrm{t}$ \\
\hline Linalool & 1091 & 1095 & 0.90 & 1.20 & 0.63 & 1.12 & 0.67 \\
\hline Terpinen-4-ol & 1179 & 1174 & 0.18 & 0.35 & - & $\mathrm{t}$ & 0.28 \\
\hline$\alpha$-Terpineol & 1192 & 1186 & 0.55 & - & 0.46 & $\mathrm{t}$ & 0.84 \\
\hline Verbenone & 1203 & 1204 & 3.00 & 0.44 & 0.27 & 0.64 & 2.00 \\
\hline Citronellol & 1214 & 1223 & 0.91 & $\mathrm{t}$ & 0.33 & 0.37 & 0.69 \\
\hline$m$-Cumenol & 1221 & 1224 & 0.37 & 0.52 & 0.82 & 0.11 & 0.59 \\
\hline Nerol & 1224 & 1227 & 1.02 & - & 0.96 & $\mathrm{t}$ & 1.81 \\
\hline Neryl acetate & 1351 & 1359 & 0.24 & 0.49 & $\mathrm{t}$ & 0.41 & 0.59 \\
\hline Isoledene & 1360 & 1374 & 1.82 & $\mathrm{t}$ & $\mathrm{t}$ & 1.67 & 4.00 \\
\hline Geranyl acetate & 1368 & 1379 & 3.58 & 2.12 & 3.12 & 2.01 & 1.09 \\
\hline$\beta$-Panasinsene & 1376 & 1381 & 1.2 & $\mathrm{t}$ & - & $\mathrm{t}$ & $\mathrm{t}$ \\
\hline$\beta$-Elemene & 1385 & 1389 & 0.29 & 0.36 & 1.06 & 0.48 & 0.57 \\
\hline iso-Caryophyllene & 1400 & 1408 & 0.65 & 0.37 & 0.27 & $\mathrm{t}$ & 0.40 \\
\hline cis-Thujopsene & 1426 & 1429 & $\mathrm{t}$ & 0.34 & 0.34 & $\mathrm{t}$ & $\mathrm{t}$ \\
\hline$\gamma$-Gurjunene & 1476 & 1475 & 0.56 & 0.42 & 0.42 & 1.11 & 1.88 \\
\hline$\gamma$-Himachalene & 1479 & 1481 & 1.36 & - & $\mathrm{t}$ & 0.40 & $\mathrm{t}$ \\
\hline$\delta$-Cadinene & 1522 & 1522 & - & 0.58 & 0.54 & - & 0.37 \\
\hline Germacrene B & 1551 & 1559 & 1.05 & $\mathrm{t}$ & - & 0.20 & 0.84 \\
\hline Total number of constituents & - & - & 31 & 32 & 28 & 30 & 30 \\
\hline Constituents identified, (\%) & - & - & $24,(98.00)$ & 22, (99.28) & $22,(99.55)$ & $24,(99.21)$ & $25,(98.33)$ \\
\hline Monoterpene hydrocarbons (\%) & - & - & 78.66 & 89.25 & 90.09 & 89.53 & 81.71 \\
\hline Oxygenated monoterpenes $(\%)$ & - & - & 8.83 & 5.84 & 3.71 & 5.82 & 8.56 \\
\hline Sesquiterpene hydrocarbons (\%) & - & - & 10.51 & 4.19 & 5.75 & 3.86 & 8.06 \\
\hline
\end{tabular}


TABLE-2

COMPARATIVE CHEMICAL PROFILES OF THE LEAF ESSENTIAL OILS OF FIVE Citrus maxima ACCESSIONS FROM SOUTH INDIA

\begin{tabular}{|c|c|c|c|c|c|c|c|}
\hline Constituent & LRI (cal.) & LRI (lit.) & $\begin{array}{c}\text { CM-TH-1 } \\
(\%)\end{array}$ & $\begin{array}{c}\text { CM-KT-2 } \\
(\%)\end{array}$ & $\begin{array}{c}\text { CM-PT-3 } \\
(\%)\end{array}$ & $\begin{array}{c}\text { CM-AL-4 } \\
(\%)\end{array}$ & $\begin{array}{c}\text { CM-TN-5 } \\
(\%)\end{array}$ \\
\hline$\alpha$-Pinene & 928 & 932 & $\mathrm{t}$ & 1.32 & 6.37 & 1.09 & 21.09 \\
\hline$\beta$-Pinene & 984 & 974 & 9.91 & 4.01 & $\mathrm{t}$ & 16.93 & $\mathrm{t}$ \\
\hline Myrcene & 989 & 988 & - & 0.42 & - & $\mathrm{t}$ & 5.14 \\
\hline Limonene & 1025 & 1024 & 11.71 & 5.95 & 78.45 & 5.29 & 7.72 \\
\hline$(\mathrm{Z})-\beta$-ocimene & 1031 & 1032 & $\mathrm{t}$ & 2.31 & - & 3.18 & $t$ \\
\hline$\alpha$-Terpinene & 1010 & 1014 & $\mathrm{t}$ & $\mathrm{t}$ & 0.14 & - & 1.67 \\
\hline Linalool & 1099 & 1095 & 4.38 & 1.19 & 1.34 & 2.16 & 1.68 \\
\hline Citronellal & 1137 & 1148 & 1.99 & 0.38 & $\mathrm{t}$ & $\mathrm{t}$ & 0.48 \\
\hline Terpinen-4-ol & 1169 & 1174 & $\mathrm{t}$ & - & 0.72 & $\mathrm{t}$ & 1.05 \\
\hline E-isocitral & 1177 & 1177 & 4.66 & 2.07 & $\mathrm{t}$ & - & 2.18 \\
\hline Citronellol & 1213 & 1223 & 3.08 & 12.91 & $\mathrm{t}$ & 5.34 & 2.99 \\
\hline cis-Carveol & 1221 & 1226 & $\mathrm{t}$ & 1.48 & 0.24 & $\mathrm{t}$ & $\mathrm{t}$ \\
\hline Nerol & 1224 & 1227 & 2.16 & 3.51 & 0.22 & 3.2 & 1.64 \\
\hline Neral & 1238 & 1235 & $\mathrm{t}$ & 3.13 & - & $\mathrm{t}$ & $\mathrm{t}$ \\
\hline cis-Myrtanol & 1250 & 1250 & $\mathrm{t}$ & 3.13 & - & $\mathrm{t}$ & 1.55 \\
\hline Dimethoxy-Z-citral & 1301 & 1316 & 5.07 & 5.65 & 0.27 & 0.64 & $\mathrm{t}$ \\
\hline Citronellyl acetate & 1341 & 1350 & 1.88 & $\mathrm{t}$ & - & 0.99 & - \\
\hline Neryl acetate & 1354 & 1359 & 4.73 & 8.61 & 0.42 & 2.50 & 5.73 \\
\hline Geranyl acetate & 1374 & 1379 & 16.41 & 6.46 & 1.49 & 7.46 & $\mathrm{t}$ \\
\hline$\beta$-Elemene & 1383 & 1389 & $\mathrm{t}$ & 1.99 & 0.42 & $\mathrm{t}$ & - \\
\hline$\beta$-Caryophyllene & 1414 & 1417 & 2.81 & 11.06 & 1.65 & 10.03 & 3.16 \\
\hline$\alpha$-Guaiene & 1433 & 1437 & 3.72 & 1.68 & 0.19 & 1.95 & 4.67 \\
\hline$\alpha$-Himachalene & 1447 & 1449 & - & 1.00 & $\mathrm{t}$ & $\mathrm{t}$ & 1.76 \\
\hline$\alpha$-Patchoulene & 1454 & 1454 & $\mathrm{t}$ & 1.87 & 1.99 & 2.94 & 0.87 \\
\hline$(\mathrm{E}, \mathrm{E})-\alpha-$ Farnesene & 1494 & 1505 & $\mathrm{t}$ & $\mathrm{t}$ & 0.31 & 0.48 & - \\
\hline$\alpha$-Cadinene & 1529 & 1537 & $\mathrm{t}$ & $\mathrm{t}$ & $\mathrm{t}$ & $\mathrm{t}$ & 0.76 \\
\hline Silphiperfol-5-en-3-ol A & 1551 & 1557 & $\mathrm{t}$ & 0.64 & $\mathrm{t}$ & 0.75 & 0.57 \\
\hline Spathulenol & 1571 & 1577 & 12.17 & 5.71 & $\mathrm{t}$ & 18.88 & 18.82 \\
\hline epi- $\alpha$-Cadinol & 1632 & 1638 & 1.96 & 1.24 & $\mathrm{t}$ & 1.77 & $\mathrm{t}$ \\
\hline z-8-hydroxy-Linalool & 1621 & 1619 & - & 1.00 & - & 1.00 & 0.71 \\
\hline$\alpha$-Cadinol & 1645 & 1652 & 3.72 & 4.14 & $\mathrm{t}$ & 4.03 & 7.69 \\
\hline Nootkatone & 1804 & 1806 & $\mathrm{t}$ & - & 2.79 & - & $\mathrm{t}$ \\
\hline Total number of constituents & - & - & 41 & 40 & 32 & 37 & 37 \\
\hline Constituents identified, (\%) & - & - & $34,(98.46)$ & $35,(97.90)$ & 29, (98.64) & $32,(98.26)$ & $34,(99.15)$ \\
\hline Monoterpenes hydrocarbons (\%) & - & - & 21.62 & 14.01 & 84.82 & 26.49 & 33.95 \\
\hline Oxygenated monoterpenes (\%) & - & - & 49.57 & 50.41 & 6.47 & 30.50 & 23.98 \\
\hline Sesquiterpene hydrocarbons (\%) & - & - & 6.53 & 17.60 & 4.25 & 14. 92 & 11.22 \\
\hline Oxygenated sesquiterpenes $(\%)$ & - & - & 20.74 & 14.88 & 3.10 & 26.35 & 30.00 \\
\hline
\end{tabular}

C. maxima peel oils (Table-1). Isoledene (4.00\%) was a major component in Kanyakumari peel oil, but it is relatively low (trace-1.82 \%) in rest of the C. maxima accessions.

Twenty-nine to thirty-four (97.90-99.15\%) constituents in C. maxima leaf oils were identified by GC-MS (Table-2). Monoterpenes hydrocarbons, oxygenated monoterpenes, sesquiterpene hydrocarbons and oxygenated sesquiterpenes in $C$. maxima leaf oils were in the range 14.01-84.82, 6.47$50.41,4.25-17.60$ and 3.10-30.00\%, respectively. Monoterpene hydrocarbons were relatively low in most $C$. maxima leaf oils compared to peel oils. Limonene content was the highest $(78.45 \%)$ in Pathanamthitta leaf oil, but its content in the rest of the $C$. maxima leaf oils were very low (5.29-11.71 $\%$ ). Spathulenol (trace-18.88 \%), caryophyllene oxide (trace$3.04 \%)$, (E)-caryophyllene (1.65-11.06 \%), geranyl acetate (trace-16.41\%) and neryl acetate $(0.42-8.61 \%)$ were the other major components of $C$. maxima leaf oils. Spathulenol and caryophyllene oxide were only in trace amounts in leaf oil of C. maxima accession from Pathanmathitta (Table-2).

Limonene contents in C. maxima peel oils were high compared to its leaf oils. Oxygenated sesquiterpenes were not found in the peel oils, but they were 3.10-30.00\% in C. maxima leaf oils. Nootkatone, a flavour constituent in grapes ${ }^{12}$, was found in trace amounts to $2.79 \%$ in $C$. maxima leaf oils. $\alpha$-Cadinene (trace-0.76\%), ledol (0-0.71\%) and (E, E)- $\alpha$ farnesene $(0-0.48 \%)$ were also found in trace amounts in C. maxima leaf oils. Isoledene (trace-4.00\%) was found in C. maxima peel oils.

\section{Conclusion}

Chemical profiles of $C$. maxima peel and leaf oils are comparable with previous reports from various geographical locations ${ }^{2,6-10}$. Limonene was the major constituent in C. maxima peel (66.85-84.08\%) and leaf (5.29-78.45\%) oils. 
Oxygenated sesquiterpenes were not found in $C$. maxima peel oils. Nootkatone was found as a minor constituent in C. maxima leaf oils (0-2.79\%). Among leaf oils, Pathanamthitta accession showed highest monoterpene content with $78.45 \%$ limonene. Monoterpenes (mainly limonene) along with sesquiterpenes and their oxygenated derivatives in relatively minor quantities result in the flavour and aroma of Citrus volatile oils ${ }^{13}$.

\section{ACKNOWLEDGEMENTS}

The authors thank the Head, Department of Chemistry, Mar Ivanios College, Thiruvananthapuram for providing the laboratory facilities and Textile Committee, Kannur for the GC-MS analyses. The authors also acknowledged to Dr. Mathew Dan, Scientist, Plant Genetic Resources Division, Jawaharlal Nehru Tropical Botanical Garden and Research Institute, Palode, Thiruvananthapuram for suggesting the genus Citrus for present study.

\section{REFERENCES}

1. A.D. Giacomo and G.D. Giacomo, in eds.: D. Giovanni and A.D. Giacomo, Essential Oil Production, In: Medicinal and Aromatic Plants, Taylor and Francis, New York, edn. 26 (2002).
2. S.M. Njoroge, H. Koaze, P.N. Karanja and M. Sawamura, J. Agric. Food Chem., 53, 9790 (2005).

3. M. Sawamura and T. Kuriyama, J. Agric. Food Chem., 36, 567 (1988).

4. M. Sawamura, T. Tsuji and S. Kuwahara, Agric. Biol. Chem., 53, 243 (1989).

5. P. Magiatis, A.L. Skaltsounis, I. Chinou and S.A. Haroutounian, $Z$. Naturforsch C, 57, 287 (2002).

6. N. Thavanapong, $\mathrm{Ph}$. D. Thesis, The Essential Oil from Peel and Flower of Citrus maxima, p. 68 (2006).

7. N.T. Minh Tu, L.X. Thanh, A. Une, H. Ukeda and M. Sawamura, Flav. Frag. J, 17, 169 (2002).

8. Z. Yong, Q. Hong-yun, W. Li-sheng and L. Xion-min, J. Guangxi Uni. Ntrl. Sc. Edn., 657 (2004).

9. K. Hosni, N. Zahed, R. Chrif, I. Abid, W. Medfei, M. Kallel, N.B. Brahim and H. Sebei, Food Chem., 123, 1098 (2010).

10. M. Sawamura, H.S. Song, H.S. Choi, K. Sagawa and H. Ukeda, Food Sci. Technol. Res., 7, 45 (2001).

11. R.P.Adams, Identification of Essential Oil Components by Gas Chromatography/Quadrupole Mass Spectroscopy, Allured, Carol Stream (2007).

12. W.D. MacLeod and N.M. Buigues, J. Food Sci., 29, 565 (1964).

13. L. Sharon-Asa, M. Shalit, A. Frydman, E. Bar, D. Holland, E. Or, U. Lavi, E. Lewinsohn and Y. Eyal, Plant J., 36, 664 (2003). 